\title{
Performance analysis comparison of optical burst switching networks' contention resolution techniques
}

\section{Laila A. Wahab Abdullah Naji ${ }^{1}$, Ibrahim Khider Eltahir ${ }^{2}$, Mohammed Al-Shargabi ${ }^{3}$,} Adel Sallam Haider ${ }^{4}$

${ }^{1}$ Department of Communication Engineering, Faculty of Engineering and Architecture, Bahri University, Khartoum, Sudan ${ }^{2}$ Department of Electronics Engineering, College of Engineering, Sudan University of Science and Technology, Khartoum, Sudan ${ }^{3}$ Department of Information Systems, Faculty of Computer Science and Information Systems, Najran University, Najran, Saudi Arabia ${ }^{4}$ Department of Information Technology Engineering, Faculty of Engineering, Aden University, Aden, Yemen

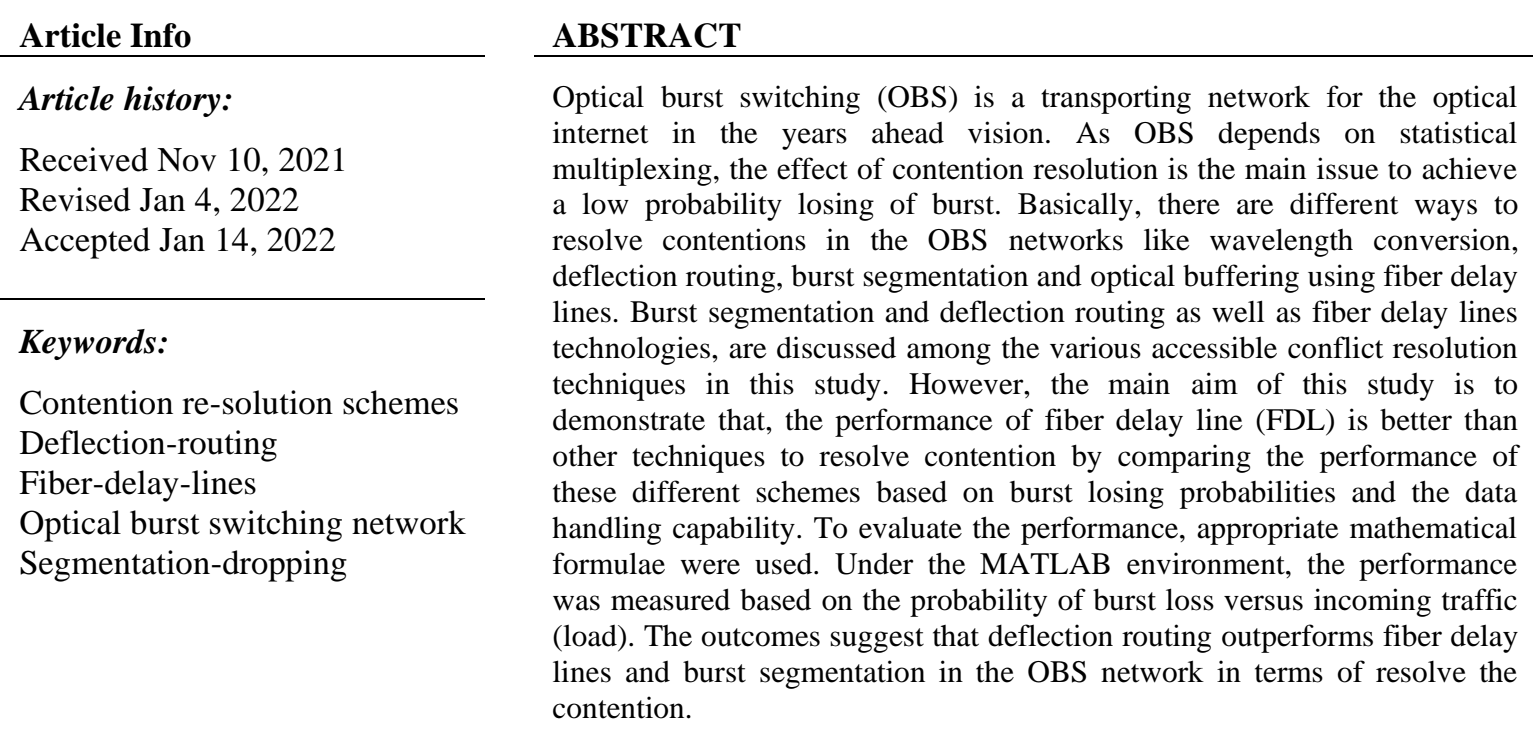

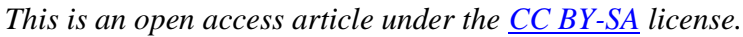

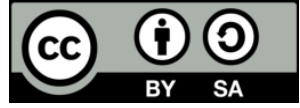

\section{Corresponding Author:}

Laila A. Wahab Abdullah Naji

Department of Communication Engineering, Faculty of Engineering and Architecture, Bahri University

Khartoum, Sudan

Email: tefke2010@gmail.com

\section{INTRODUCTION}

In the latest years, the interest for the internet has been growing, but in the present day, users rely heavily on the internet of things (IoT), artificial intelligence (AI), multimedia applications, and other internet technologies such as marketing and banking online. All of these technical advancements need a large amount of bandwidth in order to be implemented. Optical fiber may be offered solving to match the huge requirement of raw bandwidth. A single optical fiber can give a bandwidth of up to $50 \mathrm{GHz}$, so wavelength division multiplexed (WDM) is one of the solutions that matches the requirement of raw huge bandwidth [1]. WDM technology is widely used to meet the significant increase in the demand for channel capacity as a result of the rising customer and to face the challenges [2]. Optical fiber could enable the development of multimedia services due to its large bandwidth where optical fiber lines are the only way to provide bandwidth for internet services. WDM networks are used to implement bandwidth performance. Various wavelength 
channels are transported through an optical fiber line and multiplexed using WDM in optical networks. As a result, optical networks that use WDM transmission technology give a large amount of bandwidth [3].

Optical networks have used a variety of switching technologies, including circuit switching (CS), packet switching (PS), and optical burst switching (OBS) [4], [5]. Between all of them, OBS is a technology for efficient and dynamically allocating bandwidth for processing high amounts of Internet traffic [6]. In optical circuit (OC) switching, before forwarding a traffic flow, a control packet establishes a lightpath between a source-destention pair. This path is dedicated by reserved a fixed channel. The optical circuit switching (OCS) method is suitable for lengthy and consistent traffic flows, but it wasted bandwidth because it may not be fully utilized [7]. Their disadvantages take time to set up and to destroy, and those resources are not efficiently employed while the circuit is established [8] as well as the utilizing resources become hard up and the transmission of other data becomes impossible although no wasting time in waiting during switching. In OP switching, the messages are routed as individual packets in a connectionless network and there is no resource reservation, no bandwidth reservation, and no processing time scheduling for each packet. OPS, has varied implementation issues for example, connection issues that can result in information loss, delays in information delivery and optical-electronic-optical conversation [9], high cost and high power consumption, yet it can enable high bandwidth utilization [7].

OBS is a data transmission technology, which combines optical switching and transmission to convey data in large bursts between associated ingress nodes. It is created by combining the greatest aspects of both PS and optical circuit switching (CS) while avoiding their drawbacks [10]. OBS is the next evolution of optical Internet technology. It has been chosen to get better bandwidth utilization for building a flexible network that is easily for handling the burst traffic generated by multimedia services. This switching improves transferring data in optical networks with high-speed switching technology [11]. OBS consist of: control burst (CB) and data bursts (DB) [3]. Data burst and control burst are referred to burst payload and burst header packet. Both utilize separate wavelengths for transmission [12]. The CB is sent before transmitting DB by a time called an offset time, to setup a route for the data burst in the switching system [3]. Table 1 compares the three switching techniques.

Table 1. Comparison the three techniques of optical switching [5], [13]

\begin{tabular}{cccc}
\hline Characteristics/Properties & Circuit & Packet & Burst \\
\hline Bandwidth & Low & High & High \\
Set-up & High & Low & Low \\
Optical buffer & Unwanted & Wanted & Unwanted \\
Overhead Processing & Low & High & Low \\
Traffic Adaptability & Low & High & High \\
Speed's Switching & Slow & Fast & Moderate \\
Complexity Processing & Low & High & Medium \\
Signaling Scheme & Two Ways & One Way & One Way \\
\hline
\end{tabular}

\section{ARCHITECTURE OF OBS}

Optical burst switching network is bufferless in nature [14], it consists of two nodes; edge (Ingress) node and core (Egress) nodes, internet protocol (IP) packets from different clients are aggregated at the ingress in form of burst and dis-aggregated to IP packet at the egress, where they routed to their distention [15] using one way signaling resource reservation protocol [16] to passes the bursts through the network without waiting an acknowledgment from destination [14]. Figure 1 is an illustration diagram of OBS network.

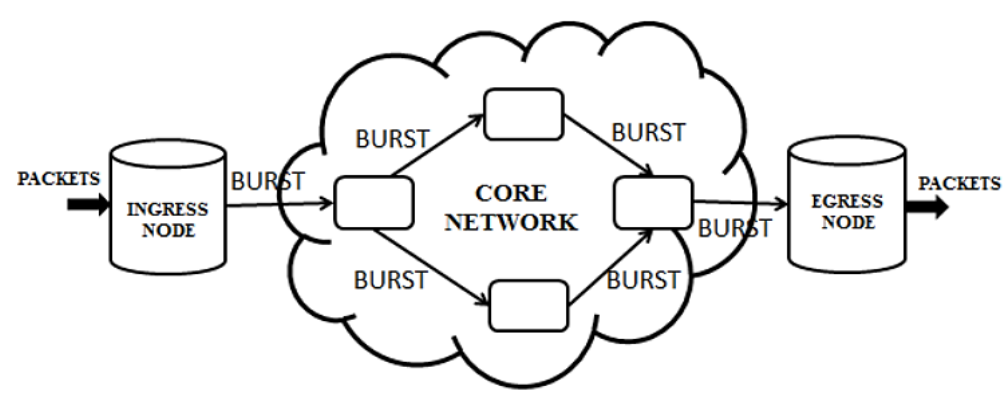

Figure 1. OBS network [9] 
The major functions of ingress nodes: assembling of burst, routing, and wavelength assignment, signaling, generating the control packet and determine the offset time. In core nodes, data bursts are shifted from one input port to other based on the information in the control packet header. Core node decides on the burst's route to resolve the contention between the numbers of bursts. Also, the major function of egress node is: disaggregates bursts to IP packets and directed the packets to the appropriate access network [15]. Inside OBS, the optical switches give a route optically over each router where no electronic data preparation takes place optically. Electronic header processing is still required in every router to obtain the switching data required to plan activities and switching. Control burst (header) is separated from the data burst and transferred in advance before the information to ensure successful preparation of the switching data and header's routing on a separated control channel [17]. The data burst switches optically without delay along its path, whereas the control packet header undergoes Optical/Electronic/Optical conversion at the intermediate nodes, which takes time for processing [5] as shown in Figure 2.

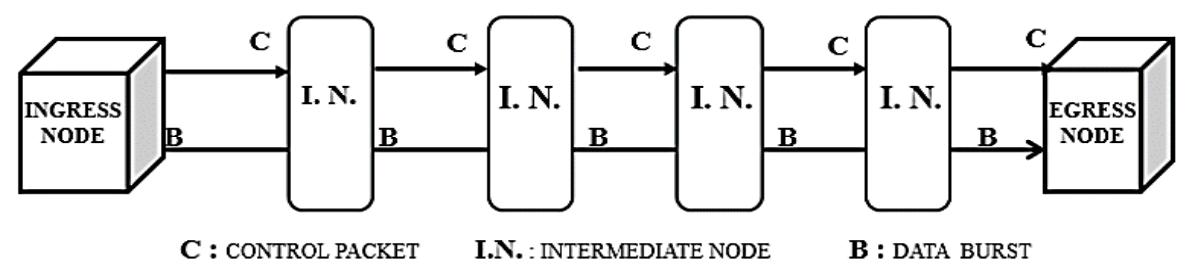

Figure 2. Diagram of burst flow [9]

When two bursts or more use the same wavelength and compete at the same time for the same output port, this will lead to contention at a core node which cause losing of data burst. Repeated losing of data burst will impact on network performance in addition to the quality of service (QoS) of the network [16]. The focus of our discussion shifts to a comparison of fiber delay lines, deflection routing, and segmentation dropping techniques. Appropriate mathematical models were constructed and simulated using MATLAB simulation to assess the contention resolving capacity of both contention resolution schemes.

This paper ordered as: In section 2 contention resolution techniques. In section 3, contention resolution by using optical buffering. In section 4 contention resolution by using segmentation. In section 5 contention resolution by using deflection routing. In section 6 simulations and results. Final conclusion in section 7 .

\section{CONTENTION RESOLUTION TECHNIQUES}

Contention is the main problem with OBS technique and has a negative impact on OBS networks. Contention occurs due to overlapping in service time [18], where the incoming burst is dropped if contention cannot resolve. It is important to resolve this problem because it is an essential performance criterion on OBS networks since it improves the quantity of service of the network [19].

Various approaches for burst contention resolution have been proposed. Optical buffering, wavelength conversion, deflection routing, and burst segmentation are the most common systems. For contention resolution between the bursts in optical buffer methods a competing burst is delay for a specific time by fiber delay lines, this delay is depending on the length of the burst. In the wavelength conversion techniques, when two or more bursts are contending for same port, one of them will directed to the adequate port while the other will directed to alternate output port by changing the wavelengths. In deflection routing, a competing burst would give a different path to its destination. Some of its disadvantages that the bursts may reach their destination out-of-order and the deflecting bursts may follow a long route to arrive their target. Bursts may have a long delay as a result of this. Furthermore, additional traffic is generated in the network when competing bursts traverse the network without needing to.

In the burst segmentation dropping scheme the burst is split into small parts called segments of burst. All of the segmented bursts are combined into one burst. When there is contention between bursts, the only segment burst those conflicts with segments of anthers burst are dropped [20]. The benefit of this technique is that it reduces the probability of burst loss without adding any additional hardware by using fragmented bursts [21]. To overcome burst losses due to contention in the network, two ways are used: reactive and proactive. The reactive contention strategy aims to resolve contentions after they exist in the core network, whereas the proactive congestion approach is aimed to avoid contentions from occurring in the network causing data burst losses [16], [22]. Figure 3 depicts the disagreement in OBS.

To resolve reactive conflict, a classic solution can be used such as: optical buffering utilizing a fiber delay lines, wavelength converter, deflecting routing and burst segmentation dropping. The only first three 
approaches are effective in resolving conflicts, but they necessitate an extra hardware and additional cost and complexity to the circuits. In the dropping segmentation strategy, the interlaced part of the burst is only discarded where the dropping will be in the tail or in the head of the burst [1].

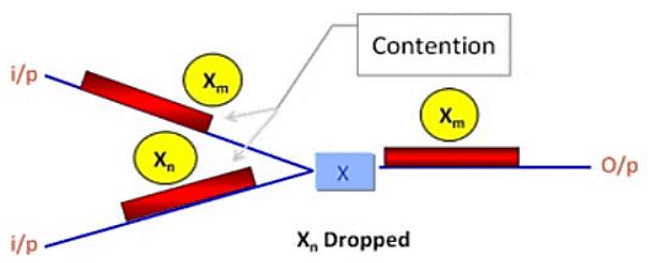

Figure 3. Contention in OBS [16], [22]

\section{CONTENTION RESOLUTION BY USING OPTICAL BUFFERING IN OBS NETWORK}

In optical buffer where risk assessment method statements (RAMs) do not available, the fiber delay line (FDLs) are used as optical buffer. Instead of being stored in the buffers, the competing bursts are delayed for a set amount of time. This done by reserving first the wavelength, then FDL reservation. At the beginning, check the availability of the wavelength where the data must wait for a minimal amount of time; if the waiting time is less than the FDL's delay, then data burst will send to FDL; otherwise, it will drop.

Before sending the data burst to FDLs it is necessary to know the FDLs's length, this length depends on burst length, which is variable in OBS so, using of buffering is limited in OBS [9]. Figure 4 illustrates a NxN OBS node and with an input port N. Assume that, the burst arrival is considered a Poisson process, the length of bursts is exponentially distributed, the mean of transmission time (mean burst length) is $1 / \mu$ and $\lambda$ is the data rate in burst per time.

Bursts from input ports are multiplexed to output ports where the received data rate at every output is $\lambda$. Assume that the interval time between bursts arrivals is represented by a random variable $t$, which is distributed exponentially with rate $\lambda$ and density of probability $\mathrm{f}_{\mathrm{T}}=\lambda e^{-\lambda t}$. A technique is presented in the node by assuming every output with a forward fixed length FDL buffering.

At out port A, before the burst reaches for it, it must specify whether there are any bursts under processing or not and also if there any bursts in the fiber delay line. The arriving burst can only export directly from port A if both FDL and port A are unused. If two bursts are contending on the same output line, one will transfer via port $\mathrm{A}$, while the other will be delayed for a while via port $\mathrm{B}$. To avoid competition between the arriving burst at port $A$ and the delaying burst over FDL, use $D=3 / \mu$. The subsequent burst packets are only discarded when there is a conflict in port B. As a result, port A's conflict already moves to port B, and the fiber delay lines delays a numerous burst before delivering them to the output port [23].

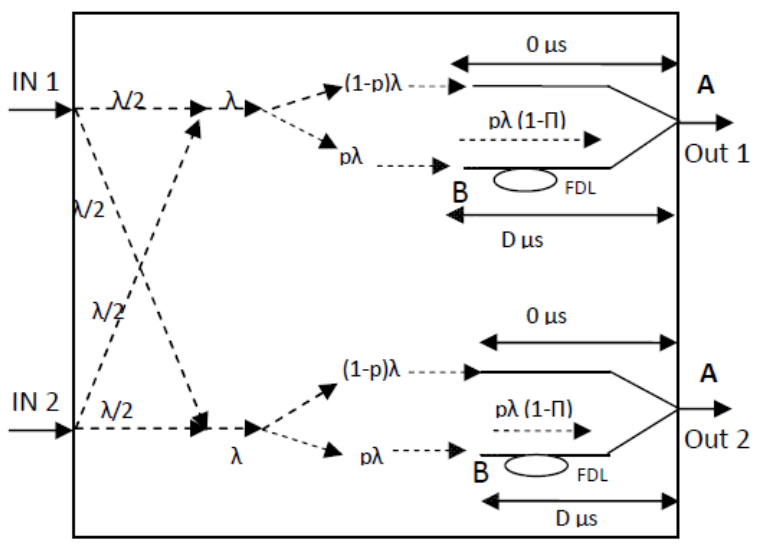

Figure 4. The arrival rates to the output FDLs from various inputs [23]

Load $(\rho)$ is the proportion of rate of arrival to the packet service rate. Assume $p$ to be the parameter that denoted to the probability of a data burst being travelling to the port B. As shown in Figure 4 , p $\lambda$ represents the arrival rate of data burst to port $\mathrm{B}$, while $\lambda(1-\mathrm{p})$ is the arrival rate of the burst to port $\mathrm{A}$. A burst 
is directly delivered to port A when there is no delay, but when the burst is delivered to port $\mathrm{B}$, means there is a delay of $\mathrm{D}$, where $\mathrm{D}$ is the FDL's propagation delay. In case, port $\mathrm{B}$ is occupied, the arriving burst from

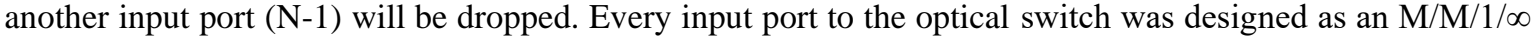
queue, in the simulation model [24], [25].

$\mathrm{N}$ is input ports for a $\mathrm{N} x \mathrm{~N}$ switching node, $\Pi$ represent the probability of port $\mathrm{B}$ when it is busy. The iterative in (1)-(6) were used to simulate the values of various network loads for $\mathrm{N}=2,4,8,16$. Then, the probability of burst loss in this output port was calculated by (7) is:

$$
\begin{aligned}
& \Phi=\frac{\mathrm{p} \lambda}{\mathrm{p} \lambda+\mu}+\frac{(1-\mathrm{p}) \lambda}{(1-\mathrm{p}) \lambda+\mu} \\
& \Pi=\frac{\mathrm{p} \lambda}{\mathrm{p} \lambda+\mu} \\
& \Gamma=\left(1-\frac{\mu}{\mu+\mathrm{p} \lambda(1-\Pi)} \mathrm{e}^{-\mathrm{p} \lambda(1-\Pi) \mathrm{D}}\right) \\
& \Psi=\left(1-\frac{\mu}{\mu+\mathrm{p} \lambda(1-\Pi)} \mathrm{e}^{-\mathrm{p} \lambda(1-\Pi) \mathrm{D}}\right) \times \frac{\mathrm{p} \lambda}{\mathrm{p} \lambda+\mu}+\frac{(1-\mathrm{p}) \lambda}{(1-\mathrm{p}) \lambda+\mu} \\
& \Omega=\frac{\lambda}{\lambda+\mu} \\
& \mathrm{p}=\Gamma+\Phi-\Psi \\
& P_{b}=\frac{\mathrm{N}}{\mathrm{N}-1} \Pi
\end{aligned}
$$

\section{OBS NETWORK CONTENTION RESOLUTION USING SEGMENTATION DROPPING}

Segmentation dropping technique resolved the conflict by discarding the overlapped part of the burst. When conflict occurs, the competing bursts are divided into segments and the overlapping ones will be dropped from the system [9]. These dropped segments can then be retransmitted again. This technique improved the throughput of the network, but still suffer from controlling the dropping segment, and drop segments regeneration [26], re-transmission and synchronization of data bursts as shown in Figure 5 [5]. Segmentation can be either segmenting the tailor as in Figure 6(a) or segmenting the header as in Figure 6(b) [26].

Head dropping has an effect on transmitting the packets in sequence, and dropping of the tail lead to give incorrect information about burst length also the header doesn't have any information about the new length because it still saves the original burst length so this indicates to ineffective utilization of the bandwidth [27]. The main difficulty in segmentation dropping technique is complex controlling and manages of drop segment and the regeneration segments. Core nodes in segmentation-based-dropping can be represented as $M / G / N_{E} W_{E}$ standard system [1], with the probability of burst loss denoted as:

$$
P=\sum_{K=n-1}^{N E W E}\left(\frac{k-n}{n} \frac{N E W E !}{k !(N E W E-k) !} \frac{\left(\frac{\lambda}{\mu}\right)^{k}}{\left(1+\frac{\lambda}{\mu}\right)^{N E W E}}\right)
$$

The bursts arrival is treated as a Poisson Process; $\lambda$ is the mean arrival rate of the burst and $\frac{1}{\mu}$ is the mean of the burst length. $\mathrm{N}_{0}$ represents the number of the output channels available, $\mathrm{W}_{0}$ is the number of available output wavelengths and $n$ is the entire of bandwidth resources, then $n=N_{0} W_{0}$. Similarly, if $N_{E}$ denotes to the available number of input channels and $\mathrm{W}_{\mathrm{E}}$ denote the number of available input wavelengths, the total available on input resource is $\mathrm{N}_{\mathrm{E}} \mathrm{W}_{\mathrm{E}}$, where $\mathrm{N}_{\mathrm{E}} \mathrm{W}_{\mathrm{E}}$ is the number of available input channels where $\mathrm{N}_{\mathrm{E}} \mathrm{W}_{\mathrm{E}} \geq \mathrm{n}[1]$.

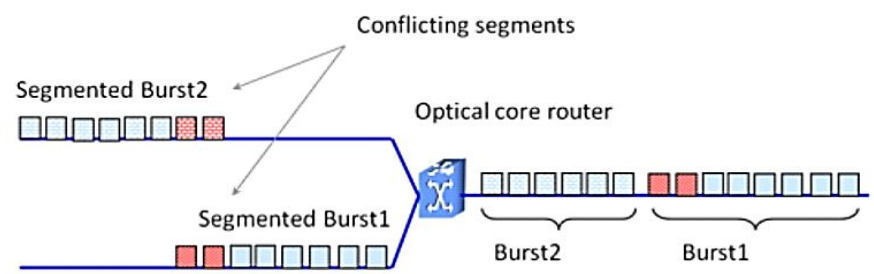

Figure 5. Segmentation dropping [9] 


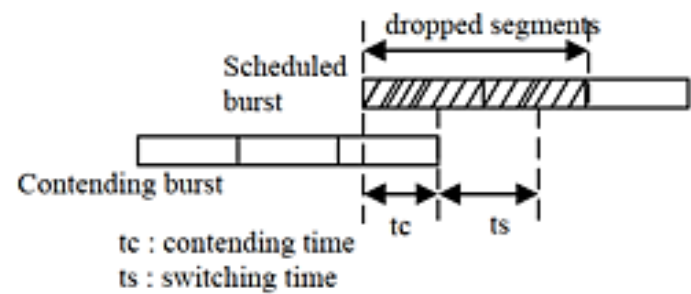

(a)

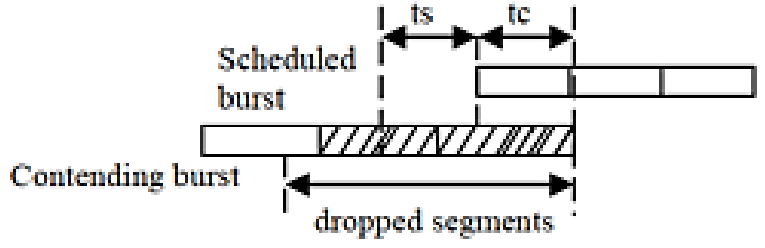

(b)

Figure 6. Segmentation dropping of (a) the tail and (b) the head [25]

\section{CONTENTION RESOLUTION BY USING DEFLECTION ROUTING IN OBS NETWORK}

Contention can be resolved by deflection routing technique by directing the competing burst to an output route other than the original port. If two data bursts are in the node $\mathrm{A}$ and want to arrive node $\mathrm{B}$ a same period of time, the shortest path from A to B will reserve for the burst that first arrive or the burst priority higher. Then, the data burst which is unprocessed will create a virtual route from $\mathrm{A}$ node to $\mathrm{B}$ over $\mathrm{C}$ to reach the nearest path to node B without wavelength conversion [1] as shown in Figure 7. So, in deflection routing, the probability of burst loss is decreasing, and utilization of the link will increase.

Congestion and re-routing of the data bursts are the main drawback in the OBS networks where the deflected burst increases congestion and it also speed-up contention and congestion on the deflection paths. When the traffic load is higher, the data burst keep-on in deviating from one route to another a few more times before reaching the required destination. This data bursts arrives out-of-order, and its effect is reflected on the data burst at the destination because the sequencing of data bursts at the egress node is important and required [9], [19]. The main limitations of deflection routing are the complexity in managing the deflected burst on the reflected route [26].

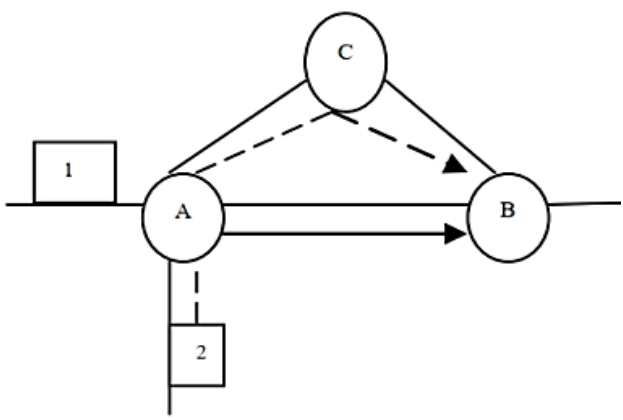

Figure 7. Principal operation of deflection routing [23]

In a deflection routing process, the probability of loss is expressed as [1], where $\mathrm{k}$ is the number of input links to the node and $\mathrm{n}$ the number of alternate paths from the source to the destination node.

$$
P=\frac{\left[\frac{\lambda}{\mu}\right]^{n} * \frac{1}{n !}}{1+\sum_{k=1}^{n}\left[\frac{\lambda}{\mu}\right]^{k} * \frac{1}{k !}}
$$

\section{SIMULATION AND RESULTS}

The capacity of the fiber delay lines, segmentation dropping and deflection routing systems to resolve contention is evaluated through simulations. Simulations were done for various of incoming traffic (load) $\left(\rho=\frac{\lambda}{\mu}\right)$ to observe the impact of $N$ (the number of wavelengths available dedicated to bursts on the output fiber links) on the burst loss probability. For different values of $\mathrm{N}$, we also looked at how the burst loss probability changed with the load.

The major issue of this paper is to prove that, the performance of FDL is better to resolve contention in comparison with other techniques. This paper compares the incoming traffics against the burst loss 
probability (BLP), whereby increasing the incoming traffics the BLP will decrease as seen in Figures 8-10. From these figures, the deflection routing technique got less BLP than other techniques, but its main drawback, during contention, the burst will deflect from one route to anther until reach to their destination, this will cause delay the burst or may lost, while in case of FDL the delay is limited depending on the length of the burst, resulting in fiber delay lines being considered the best for reducing BLP. The simulations were carried out using MATLAB tools to estimate the burst loss probability of each fiber delay line, segmentation base dropping, and deflection routing strategies for varied network characteristics under proper node and traffic assumptions using (2), (7), (8), and (9).

In this simulation, we used the total entire of bandwidth resources $n=2,4,8,16$. Also, the available number of input channels $\mathrm{N}_{\mathrm{E}}=4$, the number of available input wavelengths $\mathrm{W}_{\mathrm{E}}=10\left(\mathrm{~N}_{\mathrm{E}} \mathrm{W}_{\mathrm{E}}=40\right)$, the total availability of input resource $\mathrm{N}_{\mathrm{E}} \mathrm{W}_{\mathrm{E}} \geq \mathrm{n}$. Mean burst arrival rate $\lambda$ from 0 to 5 packets/sec, mean burst length $\frac{1}{\mu}=1 \mathrm{sec} /$ packet, load range from 0 to $5\left(\rho=\frac{\lambda}{\mu}\right)$, transmission rate $10 \mathrm{Gbps}$ and $\mathrm{D}=3 / \mu$, where $\mathrm{D}$ is the FDL's propagation delay.

Figure 8 represents the probability of burst loss versus load for various values of output channels available by fixing the number of input channel available in the segmentation dropping scheme. Also, it shows that, when the number of output channels is increased, the probability of dropping a burst is decreasing and the result validates reality. Figure 9 depicts the burst loss probability in deflecting routing schemes for various output channels values. On each output link in Deflection routing, there are W wavelengths available. Only N of the $\mathrm{W}$ output lines are dedicated to deflected bursts. Moreover, when the number of input channel keeping fixed so increasing in the number of output channels leads to decreases the burst loss probability.

Figure 10 represents the burst loss probability of fiber delay lines schemes for various values of available output channels. Obviously from the figure that increasing in the output channels causing increases in the burst loss probability. From Figures 8-10 we can conclude that: i) Deflection routing and segmentation dropping have the same principle that, reducing bursts losing probability as the number of output channels is increasing, but for fiber delay lines the increasing in number of output channels cause increasing in the burst loss probability. ii) For example, when $n=4$, the burst loss probability in segmentation dropping and deflection routing is $0.8585,0.1373$ respectively, and in fiber delay lines is 0.5327 . We can conclude that contention resolution using deflection routing is better than the other techniques. iii) It is easy to reduce burst loss probability if the network load is low, on the other hand, contention can be increased when the network load increasing. For this, increasing in burst loss probability leads to drop the OBS networks efficiency.

Finally, Figure 11 shows the comparative performance analysis of fiber delay lines, deflection routing and segmentation dropping, for various inputs and output parameters. it also shows, the deflection routing scheme outperforms the fiber delay line and segmentation dropping schemes in terms of burst loss probability of various inputs and output parameters. Because the deflection routing scheme does not delay or drop the burst like the fiber delay line and segmentation schemes do.

Figure 11 further shows that the fiber delay line is better than deflection routing because when there is conflict at any node, the burst may deflect to an alternate route, which may result the burst taking a longer route to its destination. As a result, the delay for a burst may be unacceptable, but in case of a fiber delay line, the competing bursts are delayed by a predetermined amount of time proportionate to the length of the occupied delay line. This conclusion is quite interesting and useful in terms of designing a good network with a low blocking probability.

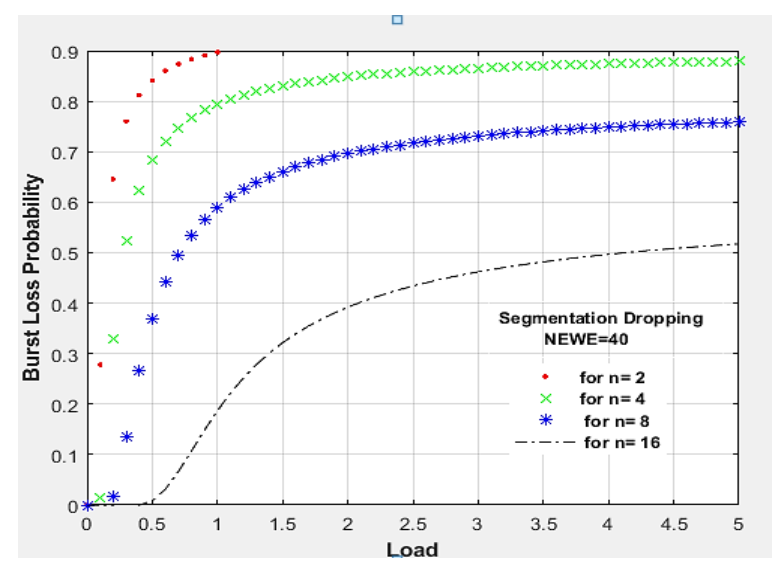

Figure 8. Burst loss probability versus incoming traffic in segmentation probability by fixing input channel while varying output channel 


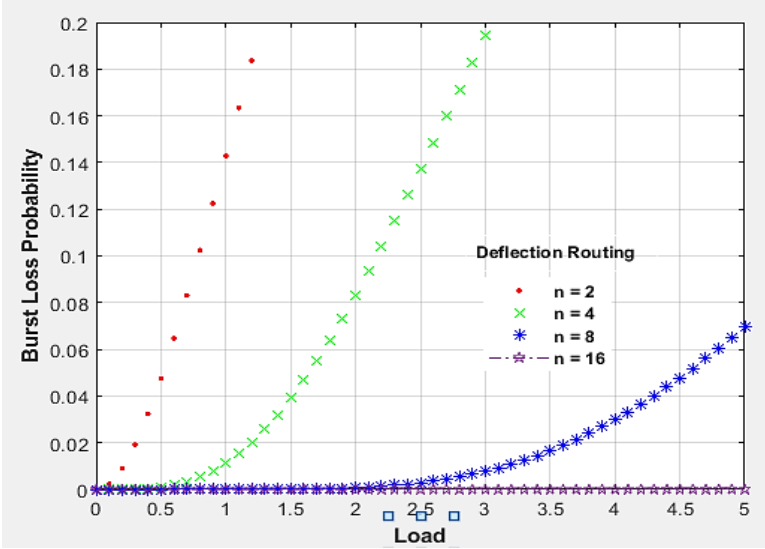

Figure 9. Burst loss probability versus incoming traffic in deflection routing by fixing input channel while varying output channel

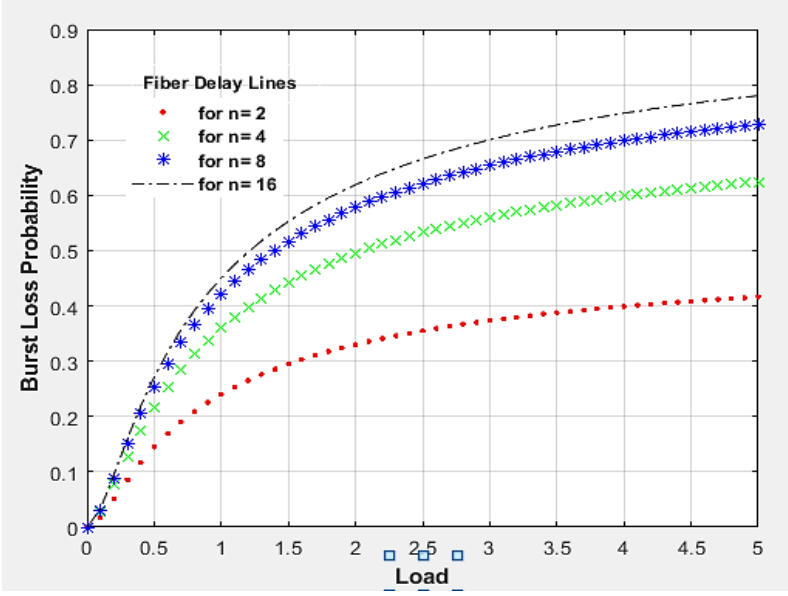

Figure 10. Burst loss probability versus incoming traffic in fiber delay lines by fixing input channel while varying output channel

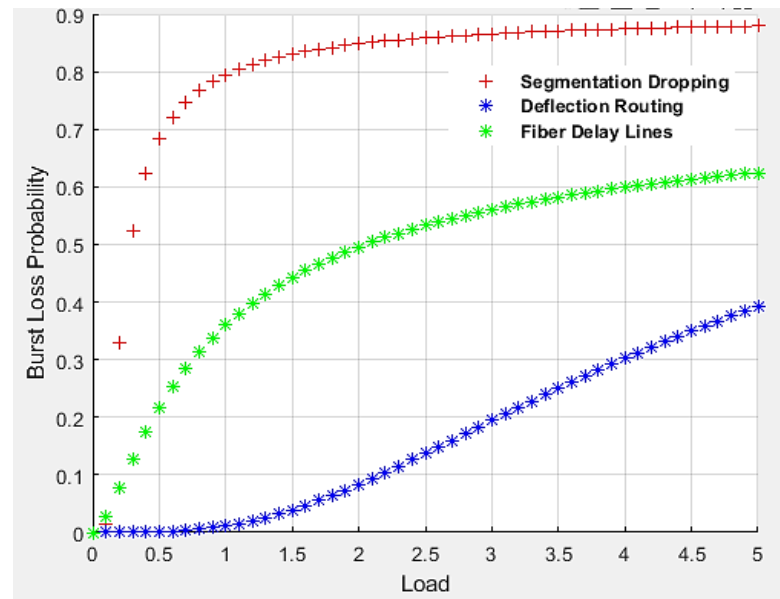

Figure 11. Comparative of the segmentation dropping, deflection routing, and fiber delay lines techniques for input channel 40 and output channel 4, respectively 


\section{CONCLUSION}

Optical burst switching (OBS) being considered as a potential solution for the next generation of the optical Internet. OBS was chosen to improve bandwidth usage in order to build a flexible network, which accommodate the traffic bursts generated by multimedia services. Overcoming performance deterioration due to contention is one of the most challenging aspects of using an OBS network. Conflict resolution is one of the most researched issues in the OBS network. Different strategies, such as deflection routing, segmentation base dropping fiber delay line, and wavelength converters schemes, can be used to solve this problem. A comparison of the performance of fiber delay lines, deflection routing, and segmentation-based dropping schemes are described in this study. All input and output network parameter values are analyzed for performance. The results suggest that fiber delay lines are more effective at resolving contention.

\section{REFERENCES}

[1] M. K. Dutta, "Performance Analysis of Deflection Routing and Segmentation Dropping Scheme in Optical Burst Switching (OBS) Network: A Simulation Study," in Advances in Intelligent Systems and Computing, vol. 988, pp. 119-128, 2020,

[2] G. R. Kavith and T. S. Indumathi, "Novel ROADM modelling with WSS and OBS to Improve Routing Performance in Optical Network," International Journal of Electrical and Computer Engineering (IJECE), vol. 6, no. 2, p. 666, Apr. 2016, doi: 10.11591/ijece.v6i2.pp666-673.

[3] H. A. M. Harb, W. M. Gaballah, A. S. Samra, A. Abo-Taleb, and A. Marwanto, "A study of the number of wavelengths impact in the optical burst switching core node," in International Conference on Electrical Engineering, Computer Science and Informatics (EECSI), vol. 2017-Decem, Sep. 2017, pp. 1-4, doi: 10.1109/EECSI.2017.8239190.

[4] V. K. A. Kumar, K. S. Reddy, and M. G. Prasad, “Optical Burst Routing by Balanced Wavelength Allocation Under Multi-objective Quality Metrics," Wireless Personal Communications, vol. 107, no. 2, pp. 1093-1114, Jul. 2019, doi: 10.1007/s11277-019-06326-w.

[5] V. K. A. Kumar, K. S. Reddy, and M. N. G. Prasad, "Review of contemporary literature on burst assembling and routing strategies in OBS networks,” Journal of Optics (India), vol. 47, no. 3, pp. 324-331, Sep. 2018, doi: 10.1007/s12596-018-0454-1.

[6] R. Poorzare and S. Abedidarabad, "A Brief Review on the Methods that Improve Optical Burst Switching Network Performance," Journal of Optical Communications, Aug. 2019, doi: 10.1515/joc-2019-0092.

[7] L. G. Dizaji and A. G. Rahbar, "Efficient Integration of Switching Mechanisms in all-optical networks," in 2016 8th International Symposium on Telecommunications, IST 2016, Sep. 2017, pp. 40-44, doi: 10.1109/ISTEL.2016.7881778.

[8] P. Kesar and M. K. Sandhu, "Security Issues and the Energy Consumption in the Optical Burst Switched Networks," International Journal of Trend in Scientific Research and Development, vol. 3, no. 4, pp. 881-885, Jun. 2019, doi: 10.31142/ijtsrd23934.

[9] S. Dubey, S. Debnath, and R. Srivastava, "Contention resolution in optical burst switches using fiber delay line buffers," Journal of Engineering Science and Technology, vol. 12, no. 2, pp. 530-547, 2017.

[10] H. Sibanda and B. Nleya, "Model analysis of dynamic priority segmented burstification and deflection routing," in 2017 IEEE AFRICON: Science, Technology and Innovation for Africa, AFRICON 2017, Sep. 2017, pp. 325-331, doi: 10.1109/AFRCON.2017.8095503.

[11] P. T. Due, D. T. Chuong, and V. V. M. Nhat, "A model of traffic prediction based admission control in OBS nodes," in RIVF 2019 - Proceedings: 2019 IEEE-RIVF International Conference on Computing and Communication Technologies, Mar. 2019, pp. 1-6, doi: 10.1109/RIVF.2019.8713683

[12] R. Q. Shaddad, A. M. Al-Ssarary, S. A. Al-mekhlafi, M. D.Qaid, and Z. M.Farhan, "Contention Resolution of Optical Burst Switching for Data Center," in 2021 International Conference of Technology, Science and Administration (ICTSA), Mar. 2021, pp. 1-4, doi: 10.1109/ICTSA52017.2021.9406552.

[13] P. Boobalan, M. Mathimanirangan, V. V. Kumar, and M. Barakathulla, "Hybrid Optical Switching In Optical Code Division Multiplexing Networks," International Journal of Research in Engineering and Technology, vol. 3, no. 2, pp. 663-667, 2014.

[14] R. Poorzare, A. Poorzare, and S. Abedidarabad, "Improving optical burst switching networks (OBS) performance by adjusting maximum burst size and burstification time," Review of Computer Engineering Studies, vol. 5, no. 1, pp. 1-6, Mar. 2019, doi: 10.18280/rces.050101

[15] R. Awasthi, L. Singh, and A. U. Khan, "Estimation of bursts length and design of a fiber delay line based OBS router," Journal of Engineering Science and Technology, vol. 12, no. 3, pp. 834-846, 2017.

[16] P. Khumalo, B. Nleya, and A. Mutsvangwa, "Intermediate Node Buffering-Based Contention Minimization Scheme," Southern Africa Telecommunication Networks and Applications Conference (SATNAC), 2019.

[17] R. Akhter and S. P. Majumder, "Performance Evaluation of a WDM Optical Network Node with Wavelength Convertible Optical Burst Switching," in 2018 International Conference on Smart Computing and Electronic Enterprise, ICSCEE 2018, Jul. 2018, pp. 1-5, doi: 10.1109/ICSCEE.2018.8538430.

[18] R. K. Maurya, S. Petale, J. Thangaraj, and V. Priye, "Burst Contention Resolution Using Fiber Delay Management in WDM Optical Networks," Wireless Personal Communications, vol. 107, no. 2, pp. 785-796, Jul. 2019, doi: 10.1007/s11277-019-06300-6.

[19] W. A. Alzoubi, H. M. S. Abdel, and N. J. S. Abu-, "Contention Resolution Technique based on Packet Switching in Optical Burst Switched Networks," International Journal of Computer Science and Information Security, vol. 15, no. 9, pp. 79-85, 2017.

[20] O. Singh, M. R. Khare, V. Shukla, S. Sharma, R. P. And, and R. Srivastava, "Estimation of burst length in optical burst switching networks based on early release of control packet," Journal of Engineering Research (Kuwait), vol. 6, no. 1, pp. 1-14, 2018.

[21] M. K. Dutta, "Comparative performance analysis of different segmentation dropping schemes under JET based optical burst switching (OBS) paradigm," in Smart Innovation, Systems and Technologies, vol. 43, 2016, pp. 385-390.

[22] B. Nleya and A. Mutsvangwa, "A Node-Regulated Deflection Routing Framework for Contention Minimization," Journal of Computer Networks and Communications, vol. 2020, pp. 1-14, Jun. 2020, doi: 10.1155/2020/2708357.

[23] V. Chamola, M. K. Dutta, and V. K. Chaubey, "Performance analysis of optical burst switching (OBS) network using fiber delay line: A simulation approach," in Proceedings - 2012 International Conference on Communication, Information and Computing Technology, ICCICT 2012, Oct. 2012, pp. 1-4, doi: 10.1109/ICCICT.2012.6398200.

[24] A. G. Fayoumi and A. P. Jayasumana, "Performance model of an optical switch using fiber delay lines for resolving contentions," in Proceedings-Conference on Local Computer Networks, LCN, vol. 2003-Janua, 2003, pp. 178-186, doi: 10.1109/LCN.2003.1243125. 
[25] Y. Fan, H. Jinzhu, Z. Mingde, and S. Xiaohan, "A contention resolution scheme by using fiber delay lines for optical burst switching," in MAPE2005: IEEE 2005 International Symposium on Microwave, Antenna, Propagation and EMC Technologies for Wireless Communications, Proceedings, vol. 2, 2005, pp. 1287-1290, doi: 10.1109/mape.2005.1618159.

[26] H. Hatamleh, "Contention and Scheduling Algorithms in Optical Burst Switched Networks," International Journal of Computer and Information Technology, vol. 5, no. 6, 2016

[27] M. K. Dutta and V. K. Chaubey, "Comparative analysis of wavelength conversion and segmentation based dropping method as a contention resolution scheme in Optical Burst Switching (OBS) network," Procedia Engineering, vol. 30, pp. 1089-1096, 2012, doi: 10.1016/j.proeng.2012.01.967.

\section{BIOGRAPHIES OF AUTHORS}
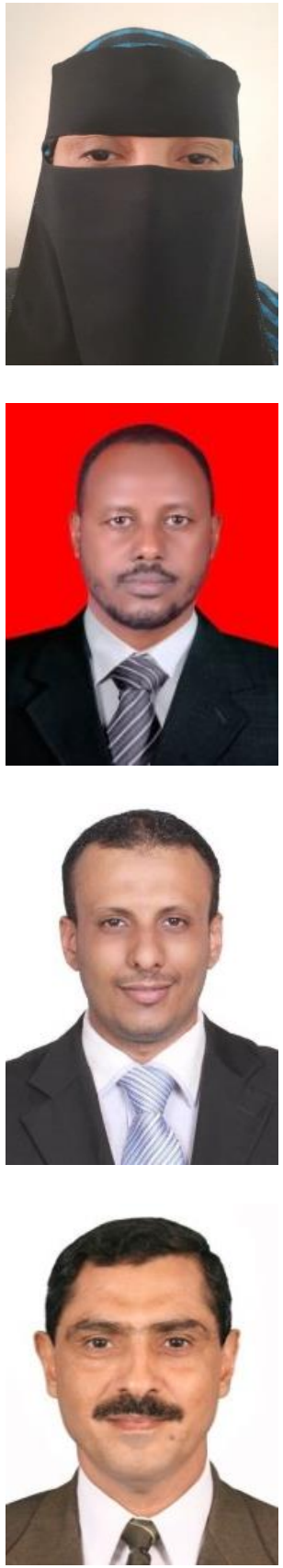

Laila A. Wahab Abdullah Naji (D) 8d SC P PhD student in Bahri University, Sudan. Received her B.Sc. Engineering degree in Electronic-Aden University-Yemen. Her M.S in Electrical-Electronics and Telecommunications from University Technology Malaysia (UTM). She can be contacted at email: tefke2010@gmail.com.

Dr. Ibrahim Khider Eltahir (iD) 8 sc P received his $\mathrm{PhD}$ in Communication Engineering and Information Systems from Huazhon University of Science and Technology. His bachelor's degree earned with first class honours at Sudan University of Science and Technology. He has over a decade of higher education teaching experience in the field of Communication Engineering. He is an associate professor at the Future University, Faculty of Telecommunication and Space. His research interest focuses on Wireless and Mobile Communication, Visible Light Communication and Software Define Network. He can be contacted at email: Ibrahim_khider@hotmail.com.

Dr. Mohammed Al-Shargabi (D) SI SC $\mathrm{P}$ received his $\mathrm{PhD}$ in Computer Networks from University Technology Malaysia, Malaysia. His Master's degree earned from Multimedia University, Malaysia. He has over a decade of higher education teaching experience in the field of Computer Networks. His research interest focuses on Quality of Service, Network Security. He can be contacted at email: mohammed218@yahoo.com.

Dr. Adel Sallam Mohamed Haider (iD 8) SC P received his B.Sc. and M.Sc. in Computer Engineering Kharkov Polytechnic Institute, Ukraine. His PhD in Technical Sciences, Saint Petersburg State Electro Technical University (LETI), Russia. Field of Specialization: Information Technology-Artificial intelligence. Professor in Information Technology. He can be contacted at email: haider.adel@gmail.com. 